\title{
SOME PROPERTIES OF ONE-DIMENSIONAL POINT INTERACTIONS
}

\author{
Saša Kočinac ${ }^{1 *}$ \\ ${ }^{1}$ Department of Physics, Faculty of Technology and Metallurgy, University of Belgrade, Belgrade, Serbia.
}

\begin{abstract}
In this review paper we shall give a brief account of some properties of so called point interactions that describe a potentials spatially localized. A different families of one-dimensional point interactions are investigated and then demonstrate how a bound state in the continuum may be generated applying supersymmetric quantum mechanics. Finally, it is shown how the latter method may be used to tailor phase rigidity of one-dimensional point interactions.
\end{abstract} relevant tunneling times are calculated. Than we

Key words: one dimensional point interactions; tunneling times; phase rigidity.

\section{INTRODUCTION}

Point interactions represent strictly localized potentials which are zero everywhere except at the origin, where suitable boundary conditions have to be imposed. An elementary and simultaneously the first example of point interaction is $\delta$ potential introduced by Fermi some 80 years ago, and ever since they have found a variety of applications in areas such as quantum mechanics (Demkov \& Ostrovskii, 1975), (Albeverio et al., 1988), solid state physics (Avron, 1994), (Exner, 1995), (Cheon et al., 2000a), (Kurasov et al., 1994), (Dzhezherya et al., 2010), (Bogolyubov et al., 2003), (Šeba, 1986) or in optics (Milanović \& Ikonić, 1997). Point interactions may be viewed as self-adjoint extensions (SAE) of kinetic energy (KE) operator. Interesting properties of this interactions such as scattering coefficients, time reversibility etc. are subject of many papers oriented both mathematically and physically (Berezin \& Faddeev, 1961), (Winful, 2006), (Carreau, 1990), (Albeverio et al.,1993), (Kurasov, 1996), (Soldatov,et al., 2006), (Cheon et al., 2000b), (Coutinho et al., 2008), (Christiansen et al., 2003).

In the first part of review, using a general approach for the calculation of tunneling times given in (Winful, 2006), we will show how an analytical expressions for the tunneling times can be derived in one-dimensional case. Then we turn our intention to some particular type of interactions and its various interpretations in literature to discuss the relevant times.

Physics
The second part deals with bound states in the continuum (BIC) generated by supersymmetry quantum mechanics (SUSY). SUSY is a method that allows one to start with any given potential and then generate a family of isospectral potentials, ie. potentials having the exact set of eigenenergies as the original one but different wave functions (Milanović, \& Ikonić, 2002), (Pappademos, et al., 1993). This allows to manipulate certain properties while retaining the original set of potential's eigenenergies. In this way it is possible to introduce bound state in continuum (BIC) on the half line of real potential (Román, \& Tarrach, 1996) or on the whole line with complex potentials (Kruchinin et al., 2010).

Third part concerns a phase rigidity of wave functions. By varying the relevant SUSY parameter $\lambda$, a shape of wave functions is also varied, thus affecting the phase rigidity of point interaction. We investigate both rigidity of continuous and bound part of the spectrum, as well as that of BIC. It turns out, however, that only phase rigidity of states from discrete part may be tailored via SUSY quantum mechanics and to this case we pay attention. For bound states in the continuum (BIC) phase rigidity is always zero, while for bound states from discrete part of spectrum phase rigidity may vary from zero to unity, depending on the strength of point interaction. 


\section{TUNNELING TIMES OF POINT INTERACTIONS}

Let $V(x)$ be an arbitrary shaped complex potential. One-dimensional Schrödinger equation describing interaction is given by:

$$
-\frac{d^{2} \psi}{d x^{2}}+V(x) \psi=E \psi
$$

In (

(1), E is particle energy, and the units are chosen such that $\hbar=1$ and $2 m=1$. The assumption is that both real and complex part of the potential extend only over the same infinitesimally small part of $x$-axes $-\varepsilon<x<+\varepsilon$. In limiting case of $\varepsilon \rightarrow 0$ potential $V(x)$ describes point interaction.. Outside the region of the interaction the general shape of the wave function that satisfies Eq.(

(1) is:

$$
\begin{aligned}
& \psi_{L}=e^{i k x}+R e^{-i k x} ;-\infty<x<-\varepsilon \\
& \psi_{\mathrm{R}}=\mathrm{Te}^{\mathrm{ikx} ;} \quad+\varepsilon<x<+\infty ;
\end{aligned}
$$

where $T=|T| \exp \left(i \varphi_{t}\right)$ and $R=|R| \exp \left(i \varphi_{r}\right)$ represent the transmission and reflection coefficients respectively, and $E=k^{2}$. As we shall see later, depending on the specific situation, shape of wave function given by (2) is not necessarily the only choice. It should be pointed out that since the potential in ( generally complex, so is the energy on the right hand side. However, the wavefuction given by (2) in that case would not be of purely oscillatory character due to complex $\kappa$ and would not account for the propagation. The tunneling times being the quantities of interest, we restrict ourselves to real energies only. Upon differentiating (

(1) with respect to energy and using its complex conjugate, the following is obtained:

$\int_{-\varepsilon}^{+\varepsilon} \psi \psi^{*} d x=\left.\left(\frac{\partial \psi}{\partial E} \frac{\partial \psi^{*}}{\partial x}-\psi^{*} \frac{\partial^{2} \psi}{\partial E \partial x}\right)\right|_{-\varepsilon} ^{+\varepsilon}+2 i \int_{-\varepsilon}^{+\varepsilon} V_{i} \psi^{*} \frac{\partial \psi}{\partial E} d x$.

In (3), $V_{i}$ stands for the imaginary part of potential $V$. Upon substituting (2) in (3), and carrying out some basic algebraic manipulations, the relationship among relevant tunneling times is derived and goes as follows:

$$
\tau_{d}=\tau_{g}-\tau_{i}+\tau_{a}
$$

For a detailed account on derivation of (4), Refs. (Zolotaryuk, 2010), (Kočinac et al., 2008) should be consulted. The left side term $\tau_{d}$ in (4) is known as dwell time and represents the total time the particle spends in the barrier of length $2 \varepsilon$, whether being transmitted or reflected:

$$
\tau_{d}=\frac{1}{k} \int_{-\varepsilon}^{\varepsilon}|\psi(x)|^{2} d x
$$

The first term on the right side of (4) is known as the bidirectional group delay $\tau_{g}$ and consists of two parts:

$$
\tau_{g}=|T|^{2} \tau_{g t}+|R|^{2} \tau_{g r}
$$

describing group delay times in transmission $\tau_{g t}$ and reflection $\tau_{g r}$ defined as:

$$
\tau_{g t}=d \varphi_{0} / d E, \quad \tau_{g r}=d \varphi_{r} / d E
$$

with $\varphi_{0}=\varphi_{t}+2 \varepsilon k$. As $\varepsilon \rightarrow 0$ phases in transmission and reflection obviously become identical $\varphi_{0}=\varphi_{t}$ due to zero length of barrier. Generally, for a barrier of finite dimension, the group delay times in transmission and reflection are different, but in a rather important case of real and symmetric barrier, it is shown that $\tau_{g t}=\tau_{g r}$. The following term, self-interference time $\tau_{i}$ is defined as follows:

$$
\tau_{i}=-\frac{1}{2 E} \operatorname{Im}(\mathrm{R})
$$

It may be viewed as a consequence of overlap of incident and reflected part of the wave function in front of the barrier, therefore interference. The last term $\tau_{a}$ in (4) is a consequence of a non-zero imaginary part of the potential and accounts for the absorption:

$$
\tau_{a}=\frac{1}{k} \int_{-\varepsilon}^{\varepsilon} V_{i} \operatorname{Im}\left(\psi^{*} \frac{\mathrm{d} \psi}{\mathrm{dE}}\right) \mathrm{dx}
$$

For a point (zero range) interaction, the dwell time (barrier time) disappears and we have $\tau_{g}=\tau_{i}+\tau_{a}$. Additionally, if the potential is real, only one relevant tunneling time remains since $\tau_{a}=0$ and Eq. (4) reduces to simple $\tau_{g}=\tau_{i}$.

Requirement for the self-adjointness of the operator $A$ yields: 


$$
\int_{-\infty}^{+\infty} \varphi^{*} A \psi d x-\int_{-\infty}^{+\infty}(A \varphi)^{*} \psi d x=0
$$

If we are dealing with kinetic energy (KE) operator $p^{2} / 2 m=-d^{2} / d x^{2}$, Eq. (10) becomes:

$$
\int_{-\infty}^{+\infty}\left(\varphi^{* \prime} \psi-\varphi^{*} \psi^{\prime \prime}\right) \mathrm{dx}=\left[\begin{array}{c}
\varphi^{*} \psi-\varphi^{*} \psi \\
{ }^{*}
\end{array}\right]_{0-}^{0+}=0
$$

When Eq. (11) equals zero, operator $A$ is self adjoint. The most obvious case for this to be fulfilled is the continuity of both wave functions $\varphi(x)$ and $\psi(x)$ and its derivatives at $x=0$, There is, however, a whole set of boundary conditions at $x=0$ that $\varphi(x)$ and $\psi(x)$ may satisfy to hold (11), although continuity of wave functions (and/or derivatives) is not fulfilled. Corresponding set of boundary conditions that satisfy (11) represent so called self-adjoint extensions (SAE) of KE operator. The general point-interactions are such SAEs whose wave functions at the origin $(x=0)$ are subject to boundary conditions of the following form:

$$
\left(\begin{array}{c}
\psi_{+} \\
\psi_{+}^{\prime}
\end{array}\right)=e^{1 \Theta}\left(\begin{array}{c}
a b \\
c d
\end{array}\right)\left(\begin{array}{c}
\psi_{-} \\
\cdot \\
\psi_{-}
\end{array}\right)=U\left(\begin{array}{c}
\psi_{-} \\
\cdot \\
\psi_{-}
\end{array}\right),
$$

where $\psi^{\prime}=d \psi / d x$ is the first derivative of the wave function with respect to $x, \psi_{ \pm}=\psi^{\prime}( \pm 0)$ and $\psi_{ \pm}=\psi( \pm 0)$. For the sake of simplicity, we will restrict ourselves to the real parameters case $(a, b, c, d, \Theta \in \Re)$. Because the wave function is determined up to a random multiplicative constant of the form $e^{1 \Theta}$, we may put $e^{1 \Theta}=1$ without losing generality (Coutinho et al., 1999). Furthermore, from Eq. (11). it follows that $a c-b d=1$ (Coutinho et al., 1999), (Christiansen et al., 2003). which finally leaves us with three independent parameters out of four $(a, b, c, d)$. Applying boundary conditions from (12) and using appropriate wave functions from (2) we can calculate coefficients $T$ and $R$ from (2) which are for obvious reasons defined as the transmission and reflection coefficients:

$$
\begin{aligned}
& T(k)=\frac{2 k}{(a+d) k+1\left(c-b k^{2}\right)} \\
& R(k)=-\frac{k(a-d)+1\left(c+b k^{2}\right)}{(a+d) k+1\left(c-b k^{2}\right)}
\end{aligned}
$$

Shape of wave function given in (2) presumes that the particle is moving from left to right. i.e. in the positive direction of $x$-axes, so called left incidence. Closer examination reveals that the simple exchange of $a$ with $d$ (and vice versa) will account for the right case incidence. Note that the transmission coefficient is invariant to the direction of the incidence. For the real potential sum of the transmission and the reflection probabilities equals one (since the potential is real there is no absorption):

$$
|T(k)|^{2}+|R(k)|^{2}=1
$$

which follows from the (13) having in mind that $a d-b c=1$. Combinations of the three independent parameters describe all SAEs of the KE operator and account for the physically important cases of point interactions. Using Eq. (13) and following the definitions in (7), we derive the following general expressions for the group times in transmission and reflection:

$$
\begin{aligned}
& \tau_{g t}=\frac{1}{2 k} \frac{(a+d)\left(c+b k^{2}\right)}{(a+d)^{2} k^{2}+\left(c-b k^{2}\right)^{2}} \\
& \tau_{g r}=\tau_{g t}+\frac{1}{2 k} \frac{(a-d)\left(b k^{2}-c\right)}{(a-d)^{2} k^{2}+\left(c+b k^{2}\right)^{2}} .
\end{aligned}
$$

Furthermore, substituting (14) and (13) in (7) one gets that the total group time is given via:

$$
\tau_{g}=\frac{1}{k} \frac{a b k^{2}+c d}{(a+d)^{2} k^{2}+\left(c-b k^{2}\right)^{2}} \text {. }
$$

The group time for the right incidence is obtained by simply interchanging $a$ and $d$ in (15). Using expression (8) for interference time, it is easily confirmed that for $a, b, c, d \in \mathfrak{R} \tau_{g}=\tau_{i}$ holds. Also note that if $a=d$, i.e. the potential is the symmetric one, than we have (Eq. (14)) $\tau_{g t}=\tau_{g r}=\tau_{g}$. In the next section we will deal with tunneling times of the specific point interactions, namely Dirac $\delta$ function and $\delta$ ' function. Regarding the latter, various interpretations of the function are still present, so we take them into consederation.

\section{1. $\delta$ function and tunneling times}

The textbook example of one-dimensional point interaction is $\delta$ function. Let the potential be of the form $V(x)=c \cdot \delta(x), c>0$ - infinite barrier. For such a barrier we have: 


$$
U=\left(\begin{array}{l}
10 \\
c 1
\end{array}\right)
$$

This can be easily verified by integrating Schrödinger equation from $-\varepsilon$ to $+\varepsilon$ and then letting $\varepsilon \rightarrow 0$, having in mind the definition of delta function itself and the definition of its integral. Due to the fact that $a=d=1$ which is consequence of delta function simmetry, there is no distinction between left and right incidence. Note that condition $a c-b d=1$ holds even for complex $c$. However, in the case of complex point interaction the relation connecting relevant times has an additional term responsible for the absorption as we already mentioned (see (4)). Putting $a=d=1, b=0$ in (13), we get

$$
T(k)=\frac{2 k}{2 k+1 c}, R(k)=\frac{-l c}{2 k+\imath c}
$$

Then, using relation for (6), we get the following expression for the group time:

$$
\tau_{g}=\frac{c}{k} \frac{1}{4 k^{2}+c^{2}}
$$

Since potential under consideration is a real and symmetric one, we have $\varphi_{0}=\varphi_{r}+\pi / 2$ and consequently $\tau_{g t}=d \varphi_{0} / d E=d \varphi_{r} / d E=\tau_{g r}$. Note that if $c<0$ in (18), then we have $\tau_{g}=\tau_{i}<0$ for all the values of particle incident energy. Also, in the case of strong $\delta$ interaction, we have $T(k)=0, R(k)=-1$. which is to be expected since in that case particle may only be reflected.

\section{2. $\delta$ ' function and tunneling times}

Next real potential to consider is $V(x)=b \delta^{\prime}(x)$ potential. As already told in introductory part, there is a great deal of controversy in literature regarding the meaning of the $\delta^{\prime}(x)$ potential. Since no final consensus on this has not been reached, we will consider both of the known interpretations of $\delta^{\prime}(x)$ potential.

In (Šeba, 1986) Šeba considered a $\delta$ function dipole potential. Roughly speaking, this interpretation of $\delta^{\prime}(x)$ treats it as if resembles a first derivative of common delta potential.

$$
V(x)=\lambda \lim _{\varepsilon \rightarrow 0} \frac{1}{2 \varepsilon^{v}}[\delta(x+\varepsilon)-\delta(x-\varepsilon)] .
$$

In the limiting case $\varepsilon k \ll 1$, the transmission and reflection coefficients become (Coutinho et al., 1999):

$$
T(k)=\frac{1 k}{\lambda^{2} \varepsilon^{1-2 v_{+1} k}} \quad R(k)=\frac{\lambda^{2} \varepsilon^{1-2 v_{k}}}{\lambda^{2} \varepsilon^{1-2 v_{+1} k}} .
$$

From the definitions of the tunneling times we have:

$$
\tau_{g}=\tau_{i}=-\frac{1}{2 E} \operatorname{Im}(\mathrm{R})=-\frac{1}{2 \mathrm{k}} \frac{\lambda^{2} \varepsilon^{1-2 v}}{\mathrm{k}^{2}+\left(\lambda^{2} \varepsilon^{1-2 v}\right)^{2}} .
$$

Both the transmission (reflection) coefficient and tunneling times depend on parameter $v$ (convergence factor) as $\varepsilon \rightarrow 0$. However, when $\varepsilon \rightarrow 0$, either $v>1 / 2$ $(T \rightarrow 0, R \rightarrow 1)$ or $v<1 / 2(T \rightarrow 1, R \rightarrow 0)$, two subspaces $x<0$ and $x<0$ are disjointed and there is no interaction; either we have total reflection or total transmission and tunneling times disappear $\tau_{g}=0$. In case that remains $v=1 / 2$, the dipole interaction case of (19) coincides with simple $\delta$ interaction with $\lambda^{2}=-c / 2$ and $\tau_{g}$ is that of (18).

In their paper, (Christiansen et al.,2003) used two different limiting procedures in approximating $\delta^{\prime}(x)$ function via :

$$
\Delta_{\varepsilon, l}(x)= \begin{cases} \pm(\varepsilon l)^{-1} & \text { for }-(\varepsilon \pm 1) / 2<\mathrm{x}<(\varepsilon \mp 1) / 2 \\ 0 & \text { elewhere }\end{cases}
$$

which are in fact two rectangles of different 'polarity' having width $\varepsilon$ and centered around $\pm l / 2$ points. Two different limiting procedures were analyzed:

$$
\delta^{\prime}(x)=\lim _{\varepsilon \rightarrow 0 l \rightarrow \varepsilon} \lim _{\varepsilon, l^{(x)}}
$$

and

$$
\delta^{\prime}(x)=\lim _{l \rightarrow 0 \varepsilon \rightarrow 0} \lim _{\varepsilon, l}(x) .
$$

Note that the latter procedure (24) is in fact the Šeba's dipole interaction described by Eq. (19) with regularizing parameter set to one $(v=1)$.

In (Christiansen et al., 2003) then showed that the first limiting procedure (243) leads to completely different transmission and reflection properties than that of Šba dipole interaction. The transmission and reflection coefficients limiting values are $T \rightarrow 0$ and $R \rightarrow-1$. Two subspaces are disjointed except for some specific values of interaction strength $\lambda_{n}$ subjected to the following condition:

$$
\tan \sqrt{\lambda}_{n}=\tanh \sqrt{\lambda}_{n}
$$

\section{Physics}


at which the $\delta(x)$ potential becomes partially transparent. For this countable set of $\lambda$ 's, both the wave function and its first derivative become discontinuous. These jumps may be described by $U$ matrix as follows:

$$
U=\left(\begin{array}{cc}
\cosh \sqrt{\lambda}_{n} / \cos \sqrt{\lambda}_{n} & 0 \\
0 & \cos \sqrt{\lambda}_{n} / \cosh \sqrt{\lambda}_{n}
\end{array}\right) .
$$

The transmission coefficient is given by: (Christiansen et al., 2003) $T=2 /(a+d)=\sec \sqrt{\lambda}_{n} \operatorname{sech} \sqrt{\lambda}_{n}$. As may be expected, due to the transparency of the potential, group time at $\lambda_{n}$ is $\tau_{g}=0$. The same follows from Eq. (13) for $b=c=0$, i.e. in the sense of tunneling times, the interaction disappears.

Previous interpretation of $\delta$ ' function leads to different values of transmission (reflection) coefficients and therefore tunneling times. Now we turn our attention to the $V(x)=b \delta^{\prime}(x)$ interaction defined by the following boundary condition (Albeverio et al., 1988), (Exner, 1996), (Winful, 2006), (Gesztesy \& Holden, 1987):

$$
U=\left(\begin{array}{c}
1 b \\
01
\end{array}\right)
$$

Here we have the continuity of wave functions first derivatives unlike in the case of $\delta$ interaction where the wave function itself is continuous. Since $a=d=1$, the group time is independent on the particle propagation direction. Thus defined $\delta$ ' is invariant under the space reflection $(x \rightarrow-x)$ in contrast to the $\delta^{\prime}(x):=d \delta(x) / d x$ definition. The relevant scattering coefficients are:

$$
T(k)=\frac{21}{b k+21}, \mathrm{R}(\mathrm{k})=\frac{\mathrm{bk}}{\mathrm{bk}+21},
$$

The group time is given by:

$$
\tau_{g}=\frac{b}{k} \frac{1}{4+k^{2} b^{2}}
$$

Just like for $\delta$ potential, $\tau_{g}$ has negative values for all particle incident energies if $b<0$. In both cases there is a bound state of energy $-\kappa^{2}$ with $\kappa^{2}=c^{2} / 4=4 / b^{2}$ for $\delta$ and $\delta$ ' potentials respectively.
3. POINT INTERACTION GENERATED BOUND STATES IN THE CONTINUUM

\subsection{Supersymmetric quantum mechanics}

Suppose that $\psi_{\varepsilon}(x)$ is a particular solution of the one-dimensional Schrödinger equation describing a particle of energy $E=\varepsilon$ moving in a real potential $U_{1}(x)$

$$
\hat{\mathrm{H}}_{1} \psi_{\varepsilon}(x)=\left[-d^{2} / d x^{2}+U_{1}(x)\right] \psi_{\varepsilon}(x)=\varepsilon \psi_{\varepsilon}(x) .
$$

One more time, the units are such that $\hbar^{2} / 2 m=1$. Knowing the particular solution of (30), the general solution for the specific energy is given as:

$$
\bar{\psi}_{\varepsilon}(x)=\psi_{\varepsilon}(x)+C \psi_{\varepsilon} \int_{x_{0}}^{x} \frac{d t}{\psi_{\varepsilon}^{2}(t)}
$$

where $x_{0}$ is real. Employing SUSY quantum mechanics (Cooper et sl., 1995) one can generate a whole family of isospectral potentials $U_{2}(x)$ having exactly the same energy spectrum as the initial potential $U_{1}(x)$. The corresponding SUSY potential is:

$$
U_{2}(\lambda, x)=U_{1}(x)-\frac{d^{2}}{d x^{2}} \ln [\lambda+I(x)]
$$

with $I(x)=\int_{x_{0}}^{x} \bar{\psi}_{\varepsilon}(t)^{2} d t$. SUSY wave function for the state of energy $E=\varepsilon$ is defined as

$$
\psi_{2 \varepsilon}(\lambda, x)=C_{2 \varepsilon} \frac{\bar{\psi}_{\varepsilon}(x)}{\lambda+I(x)}
$$

The corresponding supersymmetric wave functions for all the states of energies $E_{n} \neq \varepsilon$ is somewhat lengthy to be written down so we"ll skip it. More details can be found in (Kočinac \& Milanović, 2012b).

We state that constants $\lambda$ and $C$ in Eqs. (30) and (32) may be complex constants. In most general case $C_{2 \varepsilon}$ cannot be determined analytically, though for some specific cases an analytical expressions are obtainable. For a more detailed discussion on this subject see Ref. (Pappademos et al., 1993).

We shall now apply SUSY to manipulate wave functions and potential of point interactions. Transfer matrix connecting wave functions' first derivatives and wave function at the origin may be rewritten in a more 
convenient way (Carreau et al., 1990) (Román \& Tarrach, 1996):

$$
\left(\begin{array}{c}
-\psi^{\prime}\left(0^{-}\right) \\
\psi^{\prime}\left(0^{+}\right)
\end{array}\right)=\left(\begin{array}{cc}
\varrho^{+\alpha} & -\varrho e^{1 \Theta} \\
-\varrho e^{-1 \Theta} & \varrho^{+} \beta
\end{array}\right)\left(\begin{array}{c}
\psi\left(0^{-}\right) \\
\psi\left(0^{+}\right)
\end{array}\right) .
$$

Depending on the direction of particle propagation, we distinguish left and right incidence cases. For the particle propagating in the positive direction of $x$-axes the wave function is given by:

$$
\psi_{1}(x)= \begin{cases}e^{i k x}+R_{L} e^{-i k x} & \text { for } x<0 \\ T_{L} e^{i k x} & \text { for } x>0\end{cases}
$$

while for the right incidence we have:

$$
\psi_{2}(x)= \begin{cases}T_{R^{e}} e^{-i k x} & \text { for } x<0 \\ e^{-i k x}+R_{R} e^{i k x} & \text { for } x>0 .\end{cases}
$$

and. $T_{L(R)}$ and $R_{L(R)}$ in (34) represent transmission and reflection coefficients for the left and right incidence respectively. Taking the values of the wave function and it's first derivative at the origin, we calculate the reflection and transmission coefficients for the left and right incidence to be:

$$
\begin{aligned}
& T_{L}(k)=-\frac{21 k \varrho e^{-1 \Theta}}{\left(1 k-\varrho^{-\alpha}\right)\left(1 k-\varrho^{-} \beta\right)-\varrho^{2}}=T_{R}(k) e^{-21 \Theta} \\
& R_{L}(k)=\frac{\left(1 k+\varrho^{+\alpha}\right)(1 k-\varrho-\beta)+\varrho^{2}}{\left(1 k-\varrho^{-\alpha}\right)(1 k-\varrho-\beta)-\varrho^{2}} \\
& R_{R}(k)=\frac{\left(1 k-\varrho^{-\alpha}\right)\left(1 k+\varrho^{+} \beta\right)+\varrho^{2}}{\left(1 k-\varrho^{-\alpha}\right)\left(1 k-\varrho^{-} \beta\right)-\varrho^{2}} .
\end{aligned}
$$

The transmission coefficient for the left and right incidence differ only for the phase factor $e^{-21 \Theta}$. Furthermore, when $\alpha=\beta$ and $\Theta=0$ in (35), then we have $R_{L}=R_{R}, T_{L}=T_{R}$, i.e. the potential $U(x)$ is symmetrical and there is no distinction between left and right incidence regarding the transmission and reflection probabilities which are proportional to the square modulus of respective coefficients.

\subsection{Total reflection case}

The first case we consider is when the two subspaces $L^{2}\left(R^{-}\right)$and $L^{2}\left(R^{+}\right)$are effectively disjoint and there is no probability flowing from one half-line to the other. Formally, this situation corresponds to $Q \rightarrow 0$ in transfer matrix. The transmission coefficients disappear $\left(T_{L} \rightarrow 0, T_{R} \rightarrow 0\right.$ ), so there are only reflected parts of wave functions regardless of the incidence.

Eq. (34) can be rearranged in a more suitable form, namely:

$$
\begin{aligned}
& \psi_{1}(\mathrm{x})= \begin{cases}\sin \left(\mathrm{kx}+\phi_{0}\right) & \text { for } \mathrm{x}<0 \\
0 & \text { for } \mathrm{x}>0 ;\end{cases} \\
& \psi_{2}(\mathrm{x})= \begin{cases}0 & \text { for } \mathrm{x}<0 \\
\sin \left(\mathrm{kx}+\Theta_{0}\right) & \text { for } \mathrm{x}>0\end{cases}
\end{aligned}
$$

with $\tan \phi_{0}=-k / \alpha$ and $\tan \Theta_{0}=k / \beta$. Energy spectra corresponding to wave functions given by (36) is a continuous one, since any $k=\sqrt{E}>0$ is a satisfying solution.

However, it is possible to get a bound state in the continuum using supersymmetric quantum mechanics. This is done by deleting and then restoring a bound state with energy $\mathrm{E}=\varepsilon$ and corresponding wave function $\Psi_{\varepsilon}$. Newly generated potential has the identical energy spectrum to the original one but with modified wave functions.

We proceed as follows: for a continuous state characterized by energy $\varepsilon=\mathrm{k}^{2}$ and corresponding wave function, we perform SUSY transformation according to (31) and (33). In this case it is possible to determine constants $C$ and $C_{2 \varepsilon}$ from (30) and (32) analytically.

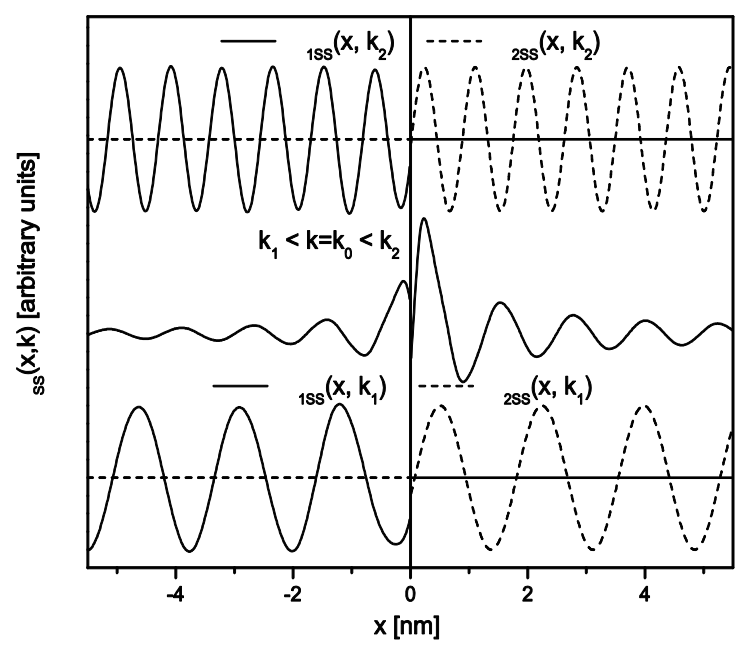

Fig. 1 Sypersymmetric wave functions at different energies for the case of total reflection $(T \rightarrow 0)$. SUSY is applied for the energy $\varepsilon_{0}=1 \mathrm{eV}$ and wave functions for energies $\varepsilon_{1}=0.5 \mathrm{eV}$ and $\varepsilon_{2}=2.0 \mathrm{eV}$ are also depicted. Parameter $\lambda$ is real and is set to 1.5. Phase factors $\tan \phi=-k / \alpha$ and $\tan \Theta=k / \beta$ are calculated for $\alpha=0.2$ and $\beta=-0.1$, in the units of $k$.

\section{Physics}




\subsection{SUSY and general point interaction}

In the previous section we have considered particle on the halfline with zero transmission $\left(T_{R(L)}=0\right)$. For the more general case of finite transmission probability, rather then employing equations (34), we use the parameterized representation of the transmission and reflection coefficients (Kočinac et al., 2012b):

$$
\begin{aligned}
& T_{L}=\cos \sigma e^{1 \tau}=T_{R} R_{L}=1 \sin \sigma e^{1(\tau+\xi)} \\
& R_{R}=1 \sin \sigma e^{1(\tau-\xi)} .
\end{aligned}
$$

Given a particle energy $E=k^{2}$, parameters $\sigma, \tau$ and $\xi$ are determined for a specific point interaction defined by $\alpha, \beta$ and $\varrho$ in transfer matrix representation (33). In that we have assumed that $\Theta$ of equals zero without losing generality in the approach.

We will simply state that unlike the case of total reflection considered in 3.2, corresponding wave functions may be presented in a form that allows an analytical derivation of SUSY wave function and potential:

$$
\begin{aligned}
& \hat{\Psi}_{1}(x, k)= \begin{cases}\cos [(k x-\xi / 2)-\omega] ; & x<0 \\
\cos [(k x-\xi / 2)+\omega] ; & x>0\end{cases} \\
& \hat{\Psi}_{2}(x, k)= \begin{cases}\sin [(k x-\xi / 2)+\omega] ; & x<0 \\
\sin [(k x-\xi / 2)-\omega] ; & x>0\end{cases}
\end{aligned}
$$

where $\omega=(\sigma+\tau) / 2$. So we are dealing with double degenerate states from continuum. Details can be found in (Kočinac et al., 2012b). Fig. 2 shows wave SUSY wave functions for a case of finite transmission.

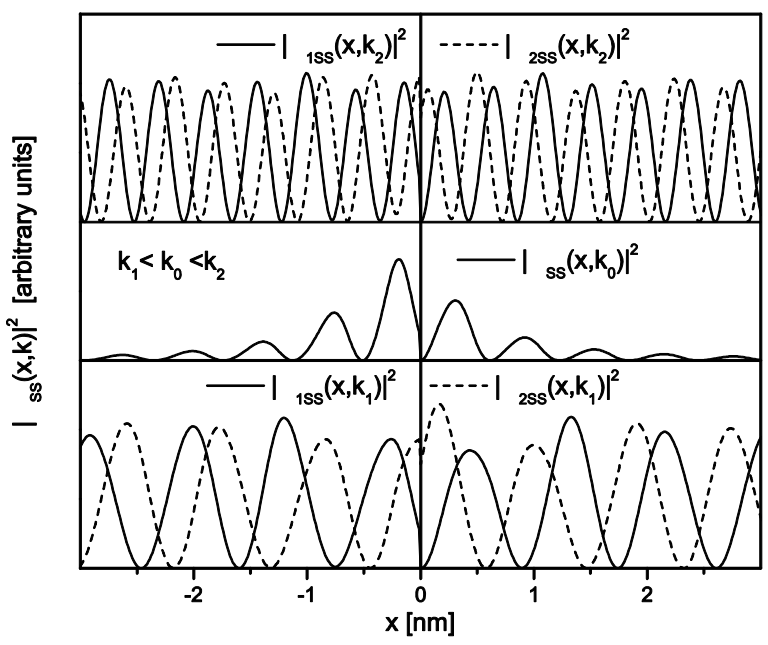

Fig. 2 Squared modulus of sypersymmetric wave functions at different energies for the case of non vanishing transmission coefficient T. SUSY is applied for the energie $\varepsilon_{0}=1 \mathrm{eV}$. Also are shown SUSY wave functions for double degenerate states at energies $\varepsilon_{1}=$ $0.5 \mathrm{eV}$ and $\varepsilon_{2}=2.0 \mathrm{eV}$. Other parameters are $\lambda=1.0+13.0$. Parameters $Q, \alpha$ and $\beta$ are $\rho=\sqrt{ } 3 / 3, \alpha=0.3$ and $\beta=-0.5$ in the units of $k$.

\section{BIORTHOGONALITY}

One more possible interpretation of transfer matrix is the one connecting wave function and its first derivative on the right and left hand side of the origin. Rearranging (33) we get (Carreau \& Gutmann,1990), (Soldatov et al., 2006):

$$
\left(\begin{array}{c}
\psi^{\prime}\left(0^{+}\right) \\
\psi\left(0^{+}\right)
\end{array}\right)=e^{-1 \Theta}\left(\begin{array}{cc}
1+\beta / \varrho & \alpha+\beta+\alpha \beta / \varrho \\
1 / \varrho & 1+\alpha / \varrho
\end{array}\right)\left(\begin{array}{c}
\psi^{\prime}\left(0^{-}\right) \\
\psi\left(0^{-}\right)
\end{array}\right)
$$

The convenience of (39) is clearly seen for limiting case $\varrho \rightarrow \infty$. All the coefficients in (39) are real with an additional restriction on $\varrho$ which is nonnegative. It can also be seen that parity of the interaction (39) is not violated as long as $\alpha=\beta$ and $\Theta=0$. There are several cases of particular interest, such as $\varrho \rightarrow 0$ corresponding to complete separation of left and right half line, and therefore no transmission between two subspaces. For $Q \rightarrow \infty$ and $\Theta=0$ Eq. (39) reduces to the well known $\delta$ interaction and so on. What we are interested in is to explore the dependence of rigidity on the initial particle energy $\varepsilon$ for the bound $(\varepsilon<0)$ states (unlike free particle whose energy is positive). In this case the wave function is defined may be defined via:

$$
\psi_{\varepsilon}(x)=\left\{\begin{array}{l}
A_{-} e^{\kappa x} \text { for } \mathrm{x}<0 \\
\mathrm{~A}_{+} \mathrm{e}^{-\kappa \mathrm{K}} \text { for } \mathrm{x}>0
\end{array}\right.
$$

where $\kappa=\sqrt{-\varepsilon}$. Upon replacing the relevant quantities in (39), the system of equations is obtained whose determinant must equal zero for the system not to have trivial solution:

$$
\left.\kappa_{1 / 2}=-Q^{-} \frac{1}{2}(\alpha+\beta) \pm \frac{1}{2} \sqrt{4 Q^{2}+(\alpha-\beta)^{2}}\right) .
$$




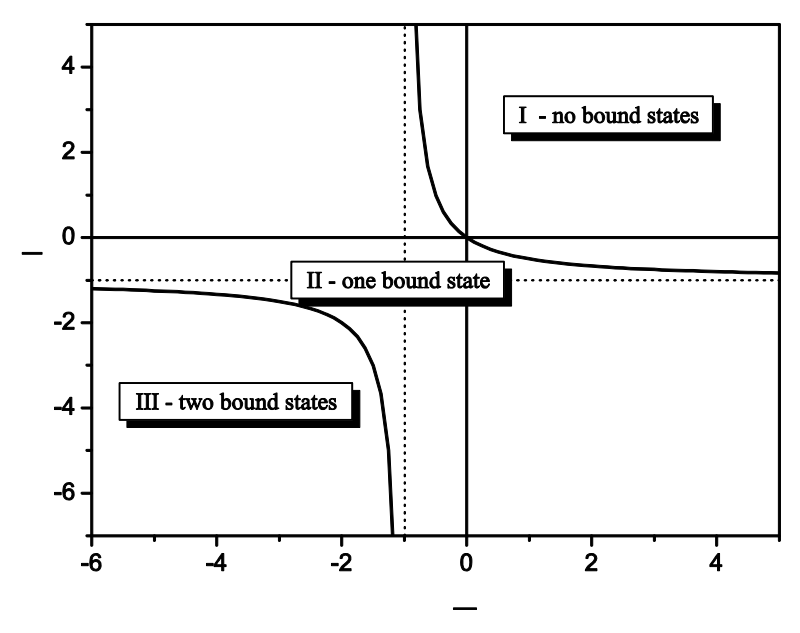

Fig. 3 Regions with two, one and no bound states are presented. Eq. (41) is rewritten in a dimensionless manner by simply dividing both sides) with $\varrho$ so that $\underline{\alpha}=\alpha / \varrho$ and $\underline{\beta}=\beta / \varrho$. The curve presented is obtained from (41) and is defined via $\underline{\beta}=-\underline{\alpha} /(1+\underline{\alpha})$.

In order to have two bound states, $\kappa_{1 / 2}$ in (4) must be positive (Fig. 3). If we have only one bound state then the upper sign is relevant in (41). For $Q=0$ only one bound state exists with energy $\sqrt{-\varepsilon}=-\alpha$ and in order to have the same eigenvalues on the left and right half-line, we must have $\alpha=\beta<0$. SUSY wave function of discrete states for specific energy $\varepsilon$ is calculated from (32):

$$
\psi_{2 \varepsilon}=\frac{A_{ \pm} e^{ \pm \kappa x}}{\lambda \pm \frac{1}{2 \kappa A_{ \pm}^{2}}\left(e^{ \pm 2 \kappa x}-1\right)}
$$

with the upper sign is used for negative part of $x$ - axes and lower for positive.

\subsection{Biorthogonality of open systems}

Systems that have non-Hermitian Hamiltonians are used to describe properties of a quantum systems that interact with environment, and are known as open quantum systems. Compared to so called closed systems that have real and orthogonal eigenvalues, this systems lack orthogonality of wave functions and interestingly enough energy eigenvalues are complex! Depending on the sign of eigenvalue imaginary part, this may lead to either decay or growth of wave function. This is overcome by the biorthogonality between wave functions.

We proceed as follows. Let us we have a complex potential:

$$
U(x)=U_{R}(x)+1 U_{I}(x) .
$$

The Schrödinger equations for two eigenvalues $E_{n}$ and $\mathrm{E}_{\mathrm{m}}$ read:

$$
\begin{aligned}
& -\psi_{\mathrm{n}}+\mathrm{U} \psi_{\mathrm{n}}=\mathrm{E}_{\mathrm{n}} \psi_{\mathrm{n}} \\
& -\psi_{\mathrm{m}}+\mathrm{U} \psi_{\mathrm{m}}=\mathrm{E}_{\mathrm{m}} \psi_{\mathrm{m}}
\end{aligned}
$$

Upon multiplying (43) with $\Psi_{\mathrm{m}}$ and (44) with $\Psi_{\mathrm{n}}$, and finally performing integration over the sum of those two, we get to:

$$
-\psi_{n} \psi_{m}+\left.\psi_{m}^{\prime} \psi_{n}\right|_{-\infty} ^{+\infty}=\left(E_{n}-E_{m}\right) \int_{-\infty}^{+\infty} \psi_{n} \psi_{m} d x
$$

If Wronskian of the wave functions $\Psi_{\mathrm{m}}$ and $\Psi_{\mathrm{n}}$

$$
W(x)=-\psi_{n}^{\prime}(x) \psi_{m}(x)+\psi_{m}^{\prime}(x) \psi_{n}(x)
$$

satisfies $W(+\infty)-W(-\infty)=0$, then from (45) it follows:

$$
\int_{-\infty}^{+\infty} \psi_{n} \psi_{m} d x=0, n \neq m
$$

and the normalization condition is:

$$
\int_{-\infty}^{+\infty} \psi_{n}^{2} d x=1, n=m
$$

Eqs. (4) and (4) are valid for all wave functions whether they belong to the discrete or continuous part of spectrum. Due to the complex nature of potential considered, we emphasize that we are dealing with non-Hermitian Hamiltonian. The normalizability condition from (4) now becomes:

$$
\int_{-\infty}^{+\infty}\left(\psi_{R}^{2}(x)-\psi_{I}^{2}(x)\right) d x=1
$$

as long as the following is fulfilled:

$$
\int_{-\infty}^{+\infty} \psi_{R}(x) \psi_{I}(x) d x=0,
$$

where $\psi_{R}$ and $\psi_{I}$ are real and imaginary part of the wave function $\psi_{n}$ respectively. If condition (Error!

Reference source not found.) is satisfied then for the standard norm (of the $n$-th eigenstate) we will have:

\section{Physics}




$$
\int_{-\infty}^{+\infty}\left(\psi_{R}^{2}+\psi_{I}^{2}\right) d x=\int_{-\infty}^{+\infty}\left|\psi_{n}\right|^{2} d x>1
$$

For a complex potential $U(x)=U_{R}+{ }_{1} U_{I}$ and single state of real eigenenergy $\varepsilon$, Eq. (Error! Reference source not found.), may be separated into two equations, i.e. rearranged in the matrix form:

$$
\left[\begin{array}{cc}
H_{0} & -U_{I} \\
U_{I} & H_{0}
\end{array}\right]\left[\begin{array}{c}
\psi_{R} \\
\psi_{I}
\end{array}\right]=\varepsilon\left[\begin{array}{c}
\psi_{R} \\
\psi_{I}
\end{array}\right]
$$

with $H_{0}=-d^{2} / d x^{2}+U_{R}$. Condition (49) needed for wave function's biorthogonality (4), (4) in general is not satisfied. However, we may "force" wave function to meet that condition. To that purpose, we construct a new wave function with $\psi_{R}$ and $\psi_{I}$ components that are related to the original $\psi_{R}$ and $\psi_{I}$ components in the following manner:

$\left[\begin{array}{c}\psi_{R} \\ \psi_{I}\end{array}\right]=\left[\begin{array}{cc}e^{1 \delta} \cos \phi & e^{1(\chi+\delta)} \sin \phi \\ e^{1 \gamma} \sin \phi & -e^{1(\chi+\gamma)} \cos \phi\end{array}\right]\left[\begin{array}{c}\psi_{R} \\ \psi_{I}\end{array}\right]=U\left[\begin{array}{c}\psi_{R} \\ \psi_{I}\end{array}\right]$

It is easy to verify that $U \cdot U^{\dagger}=I, I$ being the unitary matrix. Newly constructed wave function $\psi$ also represents eigen wave function for the state of energy $\varepsilon$. Coefficients $\phi, \chi, \gamma$ and $\delta$ in (5) are real numbers. From the definition of matrix $U$ it is clear that $e^{1 \gamma}$ and $e^{1 \delta}$ will simply be the phase factors, therefore we may put $\gamma=\delta=0$ without losing generality. Furthermore, in order for $\psi_{R}$ and $\psi_{I}$ to be real functions, we have $\chi=\pi$ (since $\chi=0$ is not the solution of (52), so Eq. (5) reduces to:

$$
\psi_{R}=\psi_{R} \cos \phi-\psi_{I} \sin \phi \psi_{I}=\psi_{R} \sin \phi+\psi_{I} \cos \phi .
$$

Equation (5) represents simple rotation of coordinate system through angle $\phi$. Now we demand wave function $\psi=A\left(\psi_{R}^{+1} \psi_{I}\right)$ to be biorthogonal, i.e.

$$
\int_{-\infty}^{+\infty} \psi_{R} \psi_{I} d x=0
$$

must hold. Constant $A$ is normalization constant of $\psi(x)$ function. Replacing (5) in ((5), angle $\phi$ that allows for biorthogonality is determined:

$$
\tan 2 \phi=\frac{-2 \int_{-\infty}^{+\infty} \psi_{R} \psi_{I} d x}{\int_{-\infty}^{+\infty} \psi_{R}^{2} d x-\int_{-\infty}^{+\infty} \psi_{I}^{2} d x}=\frac{q}{p}
$$

Biorthogonality condition now demands $\int_{-\infty}^{+\infty} \psi^{2}(x) d x=1$ and with help of simple trigonometric transformations $\cos 2 \phi=p / \sqrt{p^{2}+q^{2}}$ and $\sin 2 \phi=q / \sqrt{p^{2}+q^{2}}$, condition (Error! Reference source not found.) reduces to:

$$
A^{2}\left[\frac{p^{2}}{\sqrt{p^{2}+q^{2}}}+\frac{q^{2}}{\sqrt{p^{2}+q^{2}}}\right]=1 \Rightarrow A^{2}=\frac{1}{\sqrt{p^{2}+q^{2}}}
$$

so finally a new wave function $\psi=A\left(\psi_{R}+1 \psi_{I}\right)$ is obtained (see Eq. (26) in (Kočinac et al., 2013) finasl wzve function being normalized in the sense of (48).

\section{Phase RIGIDITY}

Phase rigidity $\varrho^{2}$ is defined as:

$$
Q=\frac{\int d r \Psi(r)^{2}}{\int d r|\Psi(r)|^{2}}
$$

With the help of (57) rigidity becomes:

$$
\varrho^{2}=\frac{\int_{-\infty}^{+\infty} \psi^{2}(x) d x}{\int_{-\infty}^{+\infty}|\psi(x)|^{2} d x}=\frac{1}{\int_{-\infty}^{+\infty}|\psi(x)|^{2} d x}=\frac{\sqrt{p^{2}+q^{2}}}{\int_{-\infty}^{+\infty}\left(\psi_{R}^{2}{ }^{2} \psi_{I}^{2}\right) d x},
$$

or finally, upon substituting $p$ and $q$ from Eq. (5), we have

$$
Q^{2}=\frac{\left[\int_{-\infty}^{+\infty}\left(\psi_{R}^{2}-\psi_{I}^{2}\right) d x\right]^{2}+\left[2 \int_{-\infty}^{+\infty} \psi_{R} \psi_{I} d x\right]^{2}}{\left[\int_{-\infty}^{+\infty}\left(\psi_{R}^{2}+\psi_{I}^{2}\right) d x\right]^{2}} .
$$

From the definition of rigidity, with the aid of CauchySchwartz inequality, it is clear that $\varrho^{2} \in(0,1]$, and 


$$
\varrho^{2}=\frac{\left(J_{1}-J_{2}\right)^{2}+\left(2 J_{3}\right)^{2}}{\left(J_{1}+J_{2}\right)^{2}}
$$

where

$$
J_{1}=\int_{-\infty}^{\infty} \psi_{R}^{2}(x) d x, J_{2}=\int_{-\infty}^{\infty} \psi_{I}^{2}(x) d x
$$

and

$$
J_{3}=\int_{-\infty}^{\infty} \psi_{R}(x) \psi_{I}(x) d x .
$$

We note one more time that in determining phase rigidity through (60) we do not need to have

$$
\int_{-\infty}^{\infty} \psi_{R}(x) \psi_{I}(x) d x=0 .
$$

\section{PHASE RIGIDITY OF SUSY MANIPULATED POINT INTERACTIONS}

We will assume that constants $C=0$ in (30) so that $\psi_{\varepsilon}(x)=\psi_{\varepsilon}(x)$. For the state of energy $E_{n}=\varepsilon$ we perform SUSY transformation.

It is crucial to emphasize at this point that state of energy $E_{n}=\varepsilon$ may belong to either continuous or discrete part of the spectrum. If $\varepsilon>0$ we have double degenerate continuous states and if $\varepsilon<0$ we have bound state(s) depending on (41). As far as rigidity is concerned, constant $C_{2 \varepsilon}$ in (32) is irrelevant, so we may put $C_{2 \varepsilon}=1$. Hence, $J_{1}, J_{2}$ and $J_{3}$ from (62) and (63) are given as:

$$
\begin{aligned}
& J_{1}=\int_{-\infty}^{\infty} \frac{\psi_{\varepsilon}^{2}\left(\lambda_{R}+I(x)\right)^{2} d x}{\left.\left(\lambda_{R}+I(x)\right)^{2}+\lambda_{I}^{2}\right]^{2}} \\
& J_{2}=\lambda_{I}^{2} \int_{-\infty}^{\infty} \frac{\psi_{\varepsilon}^{2} d x}{\left.\left[\lambda_{R}+I(x)\right)^{2}+\lambda_{I}^{2}\right]^{2}} \\
& J_{3}=-\lambda \int_{-\infty}^{\infty} \frac{\psi_{\varepsilon}^{2}\left(\lambda_{R}+I(x)\right) d x}{\left[\left(\lambda_{R}+I(x)\right)^{2}+\lambda_{I}^{2}\right]^{2}} .
\end{aligned}
$$

Integrals $J_{1}, J_{2}$ and $J_{3}$ are easily rearranged noting that $d\left(\lambda_{R}+I(x)\right)=\psi_{\varepsilon}^{2} d x$. The solution of the integrals are respectively:

\section{Physics}

Solutions of the form given in Eq. (63) are valid for both SUSY generated bound states in continuum and SYSY manipulated bound states from discrete part of the spectrum. In the most general case, expression for rigidity that is calculated from (61) with help of (64).

Asymptotic behavior of integral $I(x)$ is different for bound states and those belonging to continuous part of spectrum. If we are dealing wit bound states then we'll have $I(-\infty)=0$ and $I(+\infty)=r$, where $r$ is nonnegative.

Note that $\varrho^{2} \rightarrow 1$ when $\lambda_{I} \rightarrow 0$.

\subsection{Continuum states}

For the states from the continuum manipulated via SUSYQM (but $E_{n} \neq \varepsilon$ ) the reasoning goes as follows: Wronskian in (46) tends to zero as $x \rightarrow \pm \infty$ and the constant $C_{2 n}$ may be determined analytically in this case, i.e. $C_{2 n}=1 /\left(E_{n}-\varepsilon\right)$. Thus the SUSY wave function $\psi_{2 n}(x) \approx \psi_{1 n}(x)$ for large enough $|x|$, where the latter is the original real wave function. The contribution of imaginary part of the integral is negligible and we have $\varrho^{2} \rightarrow 1$.

\subsection{Bound states in continuum (BIC)}

For the bound state in the continuum (which may exist only if $\psi_{\varepsilon}$ belongs to the continuous part of the spectrum) the integral $I(x)$ will have a term proportional to $x$ due to the oscillatory nature of the original wave function. Therefore, we will have $I(x)(x \rightarrow \pm \infty) \rightarrow \pm \infty$, thus first terms in integrals $J_{1}$ and $J_{2}$ as well as integral $J_{3}$ disappear, leaving $J_{1}=J_{2}$ and consequently $\varrho^{2}=0 . \mathrm{T}$

\subsection{Discrete states}

If $\psi_{\varepsilon}$ is wave function of a bound state, then for all $\psi_{m}$ that belong to double degenerate state from continuum we will have $Q^{2}=1$. If there is only one bound state the SUSY wave function is calculated according to (32). If, however, there are two discrete states, then we perform SUSY for only one state of energy $E=\varepsilon$ while SUSY wave function for the remaining discrete state is done using (6) in Ref. (Kočinac, 2013), i.e: 


$$
\psi_{2 n}=\psi_{1 n}{ }^{-} \psi_{\varepsilon} \frac{\int_{-\infty}^{x} \psi_{\varepsilon} \psi_{1 n} d x}{\left(\lambda_{R}+I\right)+1 \lambda I}=\left(\psi_{2 n}\right)_{R}+\left(\psi_{2 n}\right)_{I}
$$

Here we have chosen to calculate phase rigidity for a single discrete state corresponding to region II in Fig. 3. Although analytical solution to the problem is formally possible, no reasonable expression (from which to deduce rigidity dependence on relevant interaction parameters) is obtainable. So we turn to the numerical results and vary parameter $\varrho$ (and particle energy $\varepsilon$ therefore) to demonstrate how rigidity depends on particle energy. One such dependence is presented in Fig. 4 where for different values of complex SUSY parameter $\lambda$ we have varied point interaction strength parameter $\varrho$ from (41). Phase rigidity is calculated using (58) with $\psi_{R}=\operatorname{Re}\left\{\psi_{2 \varepsilon}\right\}$ and $\psi_{I}=\operatorname{Im}\left\{\psi_{2 \varepsilon}\right\}$ where $\psi_{2 \varepsilon}$ is given by (42).

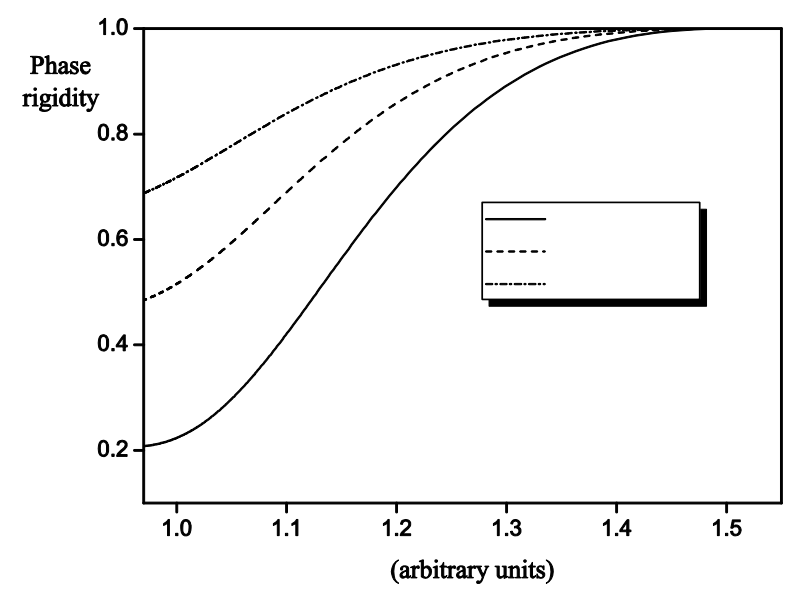

Fig. 4 Phase rigidity for SUSY manipulated bound state of general point interaction. SUSY parameter $\lambda=\left(\lambda_{\mathrm{R}}, \lambda_{\mathrm{I}}\right)$ from the top to the bottom curve is set to $(0.2,0.2),(0.15,0.15),(0.2,0.2)$ vrespectively. Parameters of point interaction (see Eq. (4)) are set to $\alpha=-1.5$ and $\beta=-0.4$. To different values of interaction parameter $\varrho$ correspond different values of parameter $\kappa=\sqrt{-\varepsilon}$, where $\varepsilon$ is the particle energy. For large values of $\kappa$ phase rigidity tends to 1 .

\section{CONCLUSION}

The aim of this review paper was to give a brief survey of some properties of one dimensional point interactions that describe spatially localized potentials.

First part deals with tunneling times. After the general relation connecting various times for the case of complex point interaction was derived, it was shown that for point interaction described by real parameters only one tunneling time is relevant. It is group time which measures the delay in the appearance of the wave packet at the front and at the end of the potential barrier. Then for some specific potential shapes tunneling times were calculated. So called point dipole interaction discussed by Šeba (Christiansen, et al., 2003) leads to either trivial case of $\tau_{\mathrm{g}}=0$ for $v \lessgtr 1 / 2$, i.e, the interaction disappears, or reduction to group time identical to that of simple $\delta$ potential apart for different interaction strength. by Christiansen et al. (Christiansen, et al., 2003) proposed different limiting procedure that leads to the partial transparency at specific interaction strengths for which $\tau_{\mathrm{g}}=0$. Various nterpretations of $\delta$ function used in (Albeverio et al., 1988), (Exner, 1996), (Gesztesy \& Holden, 1987), (Šeba, 1986). (continuous first derivative and jump in wavefunction itself) gives nontrivial $\tau_{g} \neq 0$ values of group time. For interactions defined through Eqs. (16) and (27), if the interaction strength coefficients $b$ and $c$ are negative, then the tunneling times also have negative values regardless of particle incident energy.

A bound state that may be embedded in the continuum by applying supersymmetric quantum mechanics. We used this technique to one dimensional point interactions by applying supersymmetric quantum mechanics. When dealing with the continuum on the half line, which is a case of total reflection, the SUSYQM parameterization constant $\lambda$ is allowed to be real valued thus providing normalizability of the SUSY wave function. For the general case of continuum states along the entire line (double degenerate states), only complex $\lambda$ allows for bound states in the continuum to be embedded. One possible application of the method presented here is in photonic crystals with complex dielectric constant where potentials described in the paper can be realized, thereby generating bound states in the continuum. Point interactions in two and three dimensionse to which SUSY is applied is possible extension of research presented.These problems are rather complex since one encounters divergence and renormalization in order to define proper point interactions.

Finally, a four-parameter family of onedimensional point interactions and its biorthogonal eigenfunctions, which were generated through SUSY quantum mechanics, was constructed. We proceeded by investigation of the phase rigidity of this complex

\section{Physics}


wave functions. It was demonstrated analytically that for SUSY generated bound state in continuum phase rigidity is always zero no matter what the incident particle energy was. Other states from continuum have unity rigidity, while states from discrete part of spectrum may have intermediate values depending on the particle energy.

\section{REFERENCES}

Albeverio, S. 1988. Solvable models in quantum mechanics. New York: Springer-Verlag.

Albeverio, S., Gesztesy, F. \& Holden, H. 1993. Comments on a recent note on the Schrodinger equation with a delta' -interaction. J. Phys. A: Math. Gen., 26 (15), pp. 3903-3904.

Albeverio, S., Dabrowski, L. \& Kurasov, P. 1998. Symmetries of Schrödinger Operator with Point Interactions. Lett. Math. Phys., 45 (1), pp. 33-47, doi: 10.1023/A:1007493325970.

Avron, J. E., Exner, P. \& Last, Y. 1994. Periodic Schrödinger operators with large gaps and WannierStark ladders. Phys. Rev. Lett., 72 (6), pp. 896-899.

Berezin, F. A. \& Faddeev, L. D. 1961. A remark on Schrödinger equation with a singular potential. Sov. Math. Dokl., 2, pp. 372-375.

Bogolyubov Jr., N. N. \& Kruchinin, S. 2003. Modern approach to the calculation of the correlation function in superconductivity models. Mod. Phys. Lett. B, 17, pp. 709-724.

Carreau, M., Farhi, W. \& Gutmann, S. 1990. Functional integral for a free particle in a box. Phys. Rev. D, 42, pp. 1194-1202.

Cheon, T., Exner, P. \& Šeba, P. 2000a. Wave function shredding by sparse quantum barriers. Phys. Lett. A, 277, pp. 1-6. Doi: 10.1016/S0375-9601(00)00690-3.

Cheon, T., Shigehara, T. \& Takayanagi, K. 2000b. Equivalence of local and separable realizations of discontinuity-inducing contact. J. Phys. Soc. Japan., 69, pp. 345-350.

Christiansen, P. L., Arnbak, H. C., Zolotaryuk, A. V., Ermakov, V. N. \& Gaidei, Y. B. 2003. On the existence of resonances in the transmission probability for interactions arising from derivatives of Dirac's delta function. J. Phys. A: Math. Gen., 36, pp. 7589-7600.

Cooper, F., Khare, A. \& Sukhatme, U. 1995. Supersymmetry and quantum mechanics. Phys. Rep. 251 (5-6), pp. 267-385.

Coutinho, F. A. B., Nogami,Y. \& Perez, J. F. 1999. Time-reversal aspect of the point interactions in onedimensional quantum mechanics. J. Phys. A: Math. Gen., 32 (12), pp. L133-L136.

Coutinho, F. A. B., Toyama, F. M. \& Nogami, Y. 2008. One-dimensional point interaction with three complex parameter. J. Phys. A: Math. Gen., 41 (23), p. 253306
Demkov, Y. N. \& Ostrovskii, V. N. 1975. Zero-Range Potentials and their Applications in Atomic Physics. Leningrad: Leningrad University Press.

Dzhezherya, Y., Novak, I. Y. \& Kruchinin, S. 2010. Orientational phase transitions of lattice of magnetic dots embedded in a London type superconductors. Supercond. Sci.Technol., 23 (10), pp. 105011.

Exner, P. 1995. Lattice Kronig-Penney Models. Phys. Rev. Lett., 74 (18), pp. 3503-3506.

Exner, P. 1996. Contact interactions on graph superlattices. J. Phys. A: Math. Gen., 29 (1), pp. 87 102.

Gadella, M., Negro, J. \& Nieto, L. M. 2009. Bound states and scattering coefficients of the $\mathrm{a} \delta(\mathrm{x})+$ b $\delta^{\prime}(\mathrm{x})$ potential. Phys. Lett. A, 373 (15), pp. 13101313.

Gesztesy, F. \& Holden, H. 1987. A new class of solvable models in quantum mechanics describing point interactions on the line. J. Phys. A: Math. Gen., 20 (15), pp. 5157- 5177.

Kočinac, S. Lj. S., Milanović, V., Ikonić ,Z. \& Indjin, D. 2007. Influence of nonparabolicity on tunneling times in semiconductor structures. Phys. Lett. A, 366 (1-2), pp. 130-133.

Kočinac, S. Lj. S. \& Milanović, V. (2008). Tunneling times in complex potentials. Phys. Lett. A, 372 (3), pp. 191-196.

Kočinac, S. Lj. S. \& Milanović, V. 2012a. Generalized point interaction and tunneling times. Mod. Phys. Lett. B, 26 (15), p. $1250092 . \quad$ doi: 10.1142/S021798491250092.

Kočinac, S. Lj. S. \& Milanović, V. 2012b. Bound states in continuum generated by point interaction and supersymmetric quantum mechanics. Mod. Phys. Lett. B, 26 (27), $1250177 . \quad$ doi: 10.1142/S0217984912501771.

Kočinac, S. Lj. S. \& Milanović, V. 2013. Phase Rigidity Of Point Interactions. Mod. Phys. Lett. B, 27 (1), 1350001. doi: 10.1142/S0217984913500012

Kruchinin, S., Nagao, H. \& Aono, S. 2010. Modern aspect of superconductivity: theory of superconductivity. World Scientific. ISBN9814261602.

Kurasov, P. B., Scrinzi, A. \& Elander, N. 1994. $\delta^{\prime}$ potential arising in exterior complex scaling. Phys. Rev. A, 49 (6), pp. 5095-5097.

Kurasov, P. 1996. Distribution theory for discontinuous test functions and differential operators with generalized coefficients. J. Math. Anal. Appl., 201 (1), pp. 297-323.

Marcuse, D. 1974. Theory of Dielectric Optical Waveguides. New York: Academic Press.

Milanović, V. \& Ikonić, Z. 1997. Optimization of nonlinear optical rectification in semiconductor quantum wells using the inverse spectral theory. Solid State Commun., 104 (8), pp. 445-450.

Milanović, V. \& Ikonić, Z. 2002. Supersymmetric generated complex potential with complete real spectrum. Phys. Lett. A, 293 (1-2), pp. 29-35. 
Pappademos, J., Sukhatme, U. \& Pagamenta, A. 1993. Bound states in the continuum from supersymmetric quantum mechanics. Phys. Rev. A, 48 (5), pp. 3525 3531.

Román, J. M. \& Tarrach, R. 1996. The regulated fourparameter one-dimensional point interaction. $\mathrm{J}$ Phys. A: Math. Gen., 29 (18), pp. 6073-6085.

Soldatov, V., Bogolyubov Jr, N. N. \& Kruchinin, S. P. 2006. Method of intermediate problems in the theory of Gaussian quantum dots placed in a magnetic field. Condensed Matter Physics, 9 (1), pp. 151-159.

*E-mail: skocinac@gmail.com
Šeba, P. 1986. Some remarks on the $\delta^{\prime}$-interaction in one dimension. Rep.Math. Phys., 24 (1), pp. 111120.

Winful, H. G. 2006. Tunneling time, the Hartman effect, and superluminality: A proposed resolution of an old paradox. Phys. Rep., 436 (1-2), pp. 1-69.

Zolotaryuk, V. 2010. Boundary conditions for the states with resonant tunnelling across the $\delta$ potential. Phys. Lett. A, 374 (15-16), pp. 16361641.doi:10.1016/j.physleta.2010.02.005.

\section{Physics}

